\title{
La flore bactérienne indologène aéro-anaérobie des laits pasteurisés conditionnés
}

\author{
(CAS PARTICULIER D'AEROMONAS)
}

par

\author{
Madame Louise VEILLET-PONCET
}

Pour obtenir le grade de Docteur es Sciences naturelles, Madame Louise Veillet-Poncet a présenté devant l'Université de Nancy, et soutenu publiquement le 21 décembre 1973, une thèse intitulée "La flore bactérienne indologène aéro-anaérobie des laits pasteurisés conditionnés (cas particulier d'Aeromonas) ", représentant l'important travail patiemment poursuivi dans le laboratoire de microbiologie dont Elle est responsable à l'Ecole de laiterie de la grande cité lorraine.

Après avoir rappelé l'évolution de la réglementation et des modalités de contrôle des laits pasteurisés, les phases successives de son étude personnelle sur la flore indologène aéro-anaérobie des laits pasteurisés conditionnés en Meurthe-et-Moselle ainsi que les conditions d'isolement et d'identification des souches microbiennes considérées, Madame L. Veillet-Poncet souligne, avec méthode, la taxonomie des coliformes et autres antéro-bactériacées isolés, puis étudie, définit et détermine, dans la mesure du possible, la position systématique des aeromonas, germes jusque-là ignorés en la matière : fréquence et importance des contaminations qui leur sont dues, interprétation de leur présence, effets en technologie laitière.

Devant nécessairement ici nous limiter, nous n'avons pu que choisir et reproduire, en ces pages, tout ce qui, dans ce brillant exposé, complété par 363 références bibliographiques, concerne spécialement ce genre de bactéries.

G. Thieulin.

A partir de quelques-uns de nos résultats, nous nous trouvons en présence de bactéries fermentaires, comme les coliformes, mais qui comme les Pseudomonas, possèdent cytochrome-oxydase, oxydase et cil polaire. La morphologie bacillaire, rectiligne, écarte l'éventua- 
lité de souches de Vibrio : il peut done s'agir d'Aeromonas dont nous allons confirmer le diagnostic.

Mais pour nous permettre de faire l'identification incontestée de nos souches, il est nécessaire de faire une mise au point sur la position systématique des Aeromonas qui nous amènera à nous fonder sur des critères sûrs pour établir le diagnostic de genre et d'espèces avec le maximum de certitude.

\subsubsection{Position systématique des Aeromonas}

Nomenclature : il semble bien que la première description d'Aeromonas remonte à la fin du siècle dernier, bien que la création du genre Aeromonas soit postérieure.

Tandis que Zimmerman en 1890 isole, à partir d'une eau de robinet Bacillus punctatus, Sanarelli en 1891, à partir du sang de grenouille, met en évidence Bacillus hydrophilus fuscus ; en 1896 Lehmann et Neumann décrivent une bactérie immobile élaborant un pigment brun hydrosoluble, bactérie qu'ils dénomment Bacterium salmonicida; Beijerinck, en 1900, étudiant une bactérie fermentante à cil polaire douée de pouvoir protéolytique, lui attribue le nom d'Aerobacter aerogenes.

C'est en 1936 que Kluyver et Van Niel créent le genre Aeromonas en reconnaissant comme espèce type Aeromonas liquefaciens (Beijerinck, 1900). A peu près au même moment, Sherago signale une septicémie provoquée chez de jeunes cobayes par Pseudomonas caviae comb. nov.; Schaperclaus quelques années plus tard observe des infections chez la carpe dues à Pseudomonas punctata. Dès lors, quelques chercheurs s'intéressent à ces bactéries et le nombre de publications concernant les Aeromonas va s'amplifiant. Griffin et al. définissent l'espèce Bacterium salmonicida, qui devient Aeromonas salmonicida, confirmé par Ewing et al., Leclerc, Schubert.

L'espèce Proteus hydrophilus responsable du " red-leg » chez la grenouille, étudiée par Kulp et Borden, Guthrie et Hitchner est assimilée à Aeromonas hydrophila par Stanier. Miles et Miles comparant Proteus melanovogenes isolé d'œufs gâtés et Aerobacter liquefaciens provenant de grenouilles atteintes du " red-leg » proposent d'inclure ces deux espèces dans le genre Aeromonas sous le nom d'espèce liquefaciens.

Dans la $7^{\text {e }}$ édition de la classification des bactéries de Bergey figurent quatre espèces dans le genre Aeromonas :

1) Aeromonas liquefaciens (Beijerinck, 1900), Kluyver et Van Niel, 1936.

2) Aeromonas punctata (Zimmermann, 1890), Sniesko, 1957.

3) Aeromonas hydrophila (Chester, 1901), Stanier, 1943. 
4) Aeromonas salmonicida (Lehmann et Neumann, 1896), Griffin, 1954.

Pivnick et Sabina étudiant la microbiologie d'émulsions d'huile isolent une bactérie dont les caractères sont comparables à ceux décrits par Crawford concernant Pseudomonas formicans : Pivnick et Sabina proposent alors de désigner cette bactérie sous le nom d'Aeromonas formicans Crawford, 1954. Eddy fait, en 1960-1961, une mise au point sur les Aeromonas et reconnaît trois espèces dans le genre Aeromonas:

Aeromonas liquefaciens (Beijerinck, 1900), Kluyver et Van Niel, 1936.

Aeromonas formicans (Crawford, 1954), Pivnick et Sabina, 1957.

Aeromonas salmonicida (Lehmann et Neumann, 1896), Griffin, 1954.

Au même moment Ewing et Johnson dénomment les bactéries $\mathrm{C}_{2 \pi}$. Ferguson, Aeromonas shigelloides. Leclerc, étudiant les Aeromonas isolés des eaux, reconnaît quatre espèces :

1) Aeromonas hydrophila ssp. formicans (Crawford, 1954).

2) Aeromonas dourgesi comb. nov.

Aeromonas dourgesi spp. anaerogenes comb. nov.

3) Aeromonas salmonicida (Lehmann et Neumann, 1896, Griffin, 1954.

4) Aeromonas shigelloides (Ferguson et Henderson, 1947), Ewing, 1960.

Mais en 1964, Eddy et Carpenter proposent pour les $C_{2 i}$ Ferguson la dénomination Plesiomonas shigelloides et éliminent ainsi l'espèce Aeromonas shigelloides du genre Aeoromonas. Après plusieurs années d'étude sur les Aeromonas Schubert propose dans le genre Aeromonas trois espèces comprenant des sous-espèces.

L'espèce type devient Aeromonas punctata (Zimmermann, 1896), Sniesko, 1957.

1) Aeromonas punctata (Zimmermann, 1896), Sniesko, 1957. Aeromonas punctata ssp. caviae (Scherago, 1936), Schubert, 1964.

2) Aeromonas hydrophila (Chester, 1901), Stanier, 1943. Aeromonas hydrophila ssp. anaerogenes (Schubert, 1964).

Aeromonas hydrophila ssp. proteolytica (Merkel et al., 1964), Schubert, 1969.

3) Aeromonas salmonicida (Lehmann et Neumann, 1896), Griffin, Sniesko et Friddle, 1953.

Aeromonas salmonicida ssp. achromogenes (Smith, 1963), Shubert, 1967.

Aeromonas salmonicida ssp. masoucida (Kinura, 1969). 
Malgré les très importants travaux de Schubert pour essayer de clarifier les problèmes que posent les Aeromonas en bactériologie, il faut bien reconnaître que la dénomination de ces bactéries varie avec le pays : aussi nous paraît-il opportun d'essayer de faire la synthèse des différentes dénominations utilisées dans les publications car c'est là une des difficultés à laquelle nous nous sommes heurtée au cours de nos recherches. Nous prenons pour référence les dénominations de Schubert.

1) - Aeromonas punctata (Zimmermann, 1890), Sniesko, 1957.

Bacillus punctatus (Zimmermann, 1890).

Bacterium punctatum (Lehmann et Neumann, 1896). Pseudomonas punctata (Chester, 1901).

- Aeromonas punctata ssp. caviae (Scherago, 1936), Schubert, 1964.

Pseudomonas caviae (Scherago, 1936).

Pseudomonas formicans (Crawford, 1954).

Aeromonas formicans (Crawford, 1954), Pivnick et Sabina, 1957.

Aeromonas hydrophila ssp. formicans (Crawford, 1954), Leclerc, 1962.

2) - Aeromonas hydrophila (Chester, 1901), Stanier, 1943.

Bacillus hydrophilus fuscus (Sanarelli, 1891).

Bacterium hydrophilum (Chester, 1901).

Aeromonas liquefaciens (Beijerinck, 1900), Khuyver et Van Niel, 1936.

Proteus hydrophilus (Bergey et al., 1923).

Proteus melanovogenes (Miles et Halnan, 1937).

Aeromonas dourgesi (Leclerc, 1962).

- Aeromonas hydrophila ssp. anaerogenes (Schubert, 1964). Aeromonas dourgesi ssp. anaerogenes (Leclerc, 1962).

- Aeromonas hydrophila ssp. proteolytica (Merkel et al., 1964), Schubert, 1969.

Aeromonas proteolytica (Merkel et al., 1964).

3) - Aeromonas salmonicida (Lehmann et Neumann, 1896), Griffin et al., 1953.

Bacterium salmonicida (Lehmann et Neumann, 1896).

- Aeromonas salmonicida ssp, achromogenes (Smith, 1963) Schubert, 1967).

Necromonas achromogenes (Smith, 1963).

- Aeromonas salmonicida ssp. masoucida (Kinura, 1969).

Il convient de signaler qu'il est vraisemblable qu'Aeromonas shigelloides ne figurera plus dans le genre Aeromonas : pour Eddy et Carpenter il est devenu Plesiomonas shigelloides et pour Hendrie et al. il est transféré dans le genre Vibrio.

Si au début de nos recherches nous avons adopté la nomenclature proposée par Leclerc, dans ce travail nous nous référons à celle de Schubert. 


\subsubsection{Définition du genre Aeromonas}

A partir des résultats des travaux parus depuis une quinzaine d'années, parmi lesquels essentiellement ceux de Eddy, Ewing, Leclerc et Schubert, et à partir de nos propres observations, nous pouvons considérer comme appartenant au genre Aeromonas les bactéries présentant en commun un certain nombre de caractères morphologiques, physiologiques, biochimiques et écologiques.

- Caractères morphologiques : Aeromonas se présente sous forme de bâtonnet rectiligne, plus ou moins long, mais plus fréquemment du type court, qui ne forme pas de spore, possède un système ciliaire polaire et ne prend pas la coloration de Gram.

- Caractères physiologiques : mobile ou non, Aeromonas est aéro-anaérobie facultatif, mésophile, possédant catalase, cytochromeoxydase et oxydase.

- Caractères biochimiques : Aeromonas dégrade les hydrates de carbone dans des conditions aérobies et anaérobies avec production d'acide accompagnée ou non de la production de gaz $\left(\mathrm{CO}_{2} \mathrm{Ou} \mathrm{H}_{2}\right)$.

Aeromonas n'utilise pas l'inositol.

Aeromonas élabore amylase, lécithinase, protéinase.

- Caractères écologiques : la majorité des espèces connues proviennent des eaux douce et marine et certaines espèces sont à l'origine d'infections chez les Poïkilothermes.

A cette définition correspondent 591 de nos souches : nous savons déjà qu'elles sont Gram-négatives, qu'elles possèdent un système ciliaire polaire, qu'elles cultivent à $30^{\circ} \mathrm{C}$, donc mésophiles, qu'elles possèdent catalase, cytochrome-oxydase et oxydase, qu'elles sont aéroanaérobies.

Le tableau 10 rend compte du caractère fermentaire de nos souches sur milieu de Kligler.

TABLEAU 10

Résultats des cultures sur milieu de Kligler des 591 souches possédant une catalase, cytochrome-oxydase et oxydase

\begin{tabular}{lr|c|c|c|c}
\hline & $\begin{array}{r}\text { Milieu de } \\
\text { Kligler }\end{array}$ & $\begin{array}{c}\text { Fermentation } \\
\text { du lactose } \\
\text { de souches }\end{array}$ & $\begin{array}{c}\text { Fermentation } \\
\text { du glucose }\end{array}$ & $\begin{array}{c}\text { Production } \\
\text { de gaz }\end{array}$ & $\begin{array}{c}\text { Production } \\
\text { de } \mathrm{H}_{2} \mathrm{~S}\end{array}$ \\
\hline & & + & + & - & - \\
321 & + & + & + & - \\
209 & + & + & + & - \\
32 & & + & + & \\
\hline
\end{tabular}


De plus, aucune de nos souches n'utilise l'inositol (étudiée sur milieu liquide en tube de Durham).

L'étude de la dégradation de l'amidon, du lait, de la gélatine, de la lécithine nous permet d'affirmer que toutes nos souches possèdent amylase, caséinase, gélatinase, lécithinase, mais que ces enzymes sont plus ou moins actives selon les espèces et les souches considérées, comme nous le verrons ultérieurement.

Donc ces bactéries aéro-anaérobies indologènes isolées de nos échantillons de lait pasteurisé conditionné appartiennent bien au genre Aeromonas.

La confrontation de nos résultats avec ceux de Schubert nous révèle quelques différences dont certaines méritent d'être approfondies. Si nous considérons les tests R.M. et V.P. (1) : Schubert donne comme variable le test R.M. pour la plupart des espèces ; l'utilisation du milieu de Fouad, effectivement, nous révèle dans un certain nombre de cas des tests R.M. et V.P. positifs, mais l'addition d'extrait de levures $(10 / 00)$ au milieu de Fouad nous permet d'obtenir une dissociation des deux tests en R.M. négatif et V.P. positif, et R.M. positif et V.P. négatif, ce qui facilite grandement l'identification des espèces.

L'étude des fermentations sucrées ne répond pas rigoureusement aux critères d'identification donnés par Schubert : il faut toutefois signaler que nos résultats sont toujours concordants en ce qui concerne la non utilisation de certains sucres ou alcools, xylose, dulcitol, inositol, adonitol.

Le fait que toutes nos souches d'Aeromonas fermentent le lactose nous suggère qu'il peut y avoir une relation entre ces Aeromonas et le milieu.

Il ressort de nos identifications qu'Aeromonas punctata ssp. caviae est le plus fréquemment isolé, suivi de Aeromonas hydrophila, Aeromonas punctata, Aeromonas hydrophila ssp. anaerogenes. Aeromonas hydrophila ssp. proteolytica et Aeromonas salmonicida ne sont pas isolés de nos échantillons de lait; nous ne devons pas en déduire que ces espèces ne se trouvent pas dans le lait pasteurisé : en effet, la façon, officielle, dont nous conduisons le contrôle de nos échantillons ne nous permet pas de révéler leur présence : la mise au point d'un milieu sélectif qui exalte la croissance des Aeromonas en inhibant celle des entérobactéries permettra peut-être de résoudre ce problème que nous sommes en train d'étudier.

(à suivre)

(1) Test R.M. :

Test V.P. : 